\title{
The Effects of Lacosamide in the Early Stages of Neural Tube Development in Chick Embryos
}

\author{
Beyhan Gürcü ${ }^{*}$, Tülay Oludağ Mete², Fatih Çöllü̈ ${ }^{1}$ Işsl Aydemir ${ }^{3}$, M. İbrahim Tuğlu ${ }^{4}$ \\ ${ }^{1}$ Manisa Celal Bayar University, Faculty of Science and Letters, Department of Biology, Manisa, \\ byhgurcu@gmail.com, \\ ${ }^{2}$ Manisa Celal Bayar University, School of Medicine, Gynecology and Obstetrics Department, Manisa, \\ ${ }^{3}$ Niğde Ömer Halisdemir University, School of Medicine, Histology-Embryology Department, Niğde, \\ ${ }^{4}$ Manisa Celal Bayar University, School of Medicine, Histology-Embryology Department, Manisa, \\ *Corresponding author
}

Received: 8 October 2017

Accepted: 13 February 2018

DOI: $10.18466 /$ cbayarfbe. 342317

\begin{abstract}
Antiepileptic drugs (AED) are teratogens that confer a risk of various congenital malformations including neural tube defects. Lacosamide (LCM) is a novel third-generation AED and its effects on neural tube (NT) development remain unclear. Thus, the aim of the present study was to investigate the effects of LCM in the early stages of NT development in the chicken embryo. Three different doses of LCM were applied under the embryonic disks of chicken embryos after they were incubated for 30 hours. Incubation was continued for an additional 80 hours and then all embryos were obtained for routine histology. In the LCM-treated groups, there was occlusion in the middle and ventral levels of the cavity. In the group treated with $1.60 \mathrm{mg}$ of LCM, the NT cavity was closed, the notochord exhibited deterioration, and cellular association appeared abnormal. In the groups treated with 0.12 and $0.5 \mathrm{mg}$ of LCM, the ectoderm layer surrounding the embryo was hypertrophic and the number of pyknotic cells increased in a dose-dependent manner. Thus, different doses of LCM had different effects. The results of this study demonstrated that LCM causes histopathological alterations during the neurulation stage that might ultimately result in the development of congenital defects and/or malformations.
\end{abstract}

Keywords: Lacosamide, Histopathology, Neural tube, Chick.

\section{Introduction}

Neural tube defects (NTDs) are the consequence of abnormal neurulation that results in heterogeneous and complex congenital anomalies of the central nervous system that are associated with a variety of medical, financial, and social problems [1,2]. NTDs occur in approximately 1 in 1,000 pregnancies in the United States and an estimated 300,000 newborns worldwide [3, 4]. NTDs may even lead to death during the antenatal or postnatal period $[1,2]$.

The neural tube (NT) normally closes in utero within 4 weeks of conception or 2 weeks after missed menstruation [2,3]. Although the causative mechanisms underlying NTDs remain poorly understood, genetic factors (chromosomal aberrations or translations), nutritional deficiencies, environmental causes, exposure to radiation, or a combination of these factors are known to play an important role in their manifestation $[2,3]$.

Prenatal exposure to antiepileptic drugs (AEDs) is associated with a relatively higher risk of NTD [5]. The teratogenic effects of various AEDs, such as valproic acid, sodium phenytoin, and levetiracetam have been investigated but the effects of LCM, which is a novel third-generation AED that has recently been used frequently for the treatment of partial-onset seizures, remain unclear. Its possible side effects on fetal development have been investigated previously [6] but detailed studies of its influence on NT development have yet to be performed.

Thus, the present study aimed to determine the effects of various concentrations of LCM on NT closure in the early stages of chicken embryo development.

\section{Materials and Methods}

This study was conducted with the cooperation of the Histology Department Research Laboratory of Celal Bayar University Medical School. Fertilized and specific-pathogen-free Leghorn chicken eggs supplied by the Republic of Turkey Ministry of Agriculture and Rural Affairs, Bornova Veterinary Control and Research Institute, were selected for the study and all experiments were approved by the Local Animal Care Ethics Committee of Celal Bayar University (no. 77.637.4335- 
27). Twenty-eight eggs with a mean ( \pm SD) weight of 65 $\pm 5 \mathrm{~g}$ were incubated for $30 \mathrm{~h}$ at $37.5 \pm 0.2^{\circ} \mathrm{C}$ and 60 $80 \%$ relative humidity; each egg was repositioned on its axis every $2 \mathrm{~h}$ until the LCM injection. After $30 \mathrm{~h}$ of incubation, the eggs were opened under $4 \times$ optical magnification [7-9] at Hamburger-Hamilton stage 9 [8] and rinsed with $70 \%$ ethanol. Then, a piece of adhesive tape was placed close to the egg air cavity and a small window was opened for the injections [6].

Next, the embryos were randomly separated into a control group (Group 1; $\mathrm{n}=7$ ) and three LCM-treated groups $(0.12 \mathrm{mg}$, sub-therapeutic group, $\mathrm{n}=7 ; 0.5 \mathrm{mg}$, therapeutic group, $\mathrm{n}=7$, and $1.6 \mathrm{mg}$, supra-therapeutic group, $\mathrm{n}=7$ ). Within each group, the embryonic disks were identified and the same volume of liquid was injected under each disk with a 30-gauge syringe, as described previously by Mete et al. [6]. Briefly, a solution of 12,50 , or $160 \mu \mathrm{L}$ of LCM was diluted with physiological saline to a total volume of $180 \mu \mathrm{L}$. In all groups, the eggs were closed with adhesive tape after the injection and incubation was continued for $80 \mathrm{~h}$, at which time the eggs were reopened and the embryos were dissected from the embryonic membranes, with adherence to microsurgical rules using the water-floating technique.

All embryos were placed in a $10 \%$ formalin solution for $24 \mathrm{~h}$. following dehydration process with alcohol, embryos pellucid with xylol and embedded in paraffin. Then, $5 \mu \mathrm{m}$ of sections were taken from the tissues. Sections were spread on the hot plate and mounted on glass slides, and were stained with Hematoxylin-Eosin. The samples were examined under a Leica (DM 4000B) bright field microscope, and their images were taken via an Olympus (DP 71) camera.

\section{Results}

Evaluations of the 80-h embryo sections demonstrated that the alar plaques, basal plaques, and NT were normal in the control group, and mesenchymal cells, proliferating cells, and pyknotic cells were observed in the notochord. Additionally, the NT epithelial cells and central channel cells were normal (Figure 1a).

In the LCM-treated groups, NT closure occurred but there were histological differences among the groups. More specifically, pyknotic cells were observed following treatment with $0.12 \mathrm{mg}$ of LCM and increased in a dose-dependent manner. Additionally, the proliferating cells were constructed in such a way as to form a compact structure (Figures $1 \mathrm{~b}, \mathrm{~d}$, e, and $\mathrm{f}$ ), obstructions were detected in the middle and ventral levels of the NT cavity (Figures $1 \mathrm{~b}, \mathrm{c}, \mathrm{d}$, and e), and the neural cavity closed at high doses (Figure 1f).

The notochord was mostly disrupted and had an abnormal structural appearance, although there were no obvious increases in cell numbers. In particular, the ectoderm layer that surrounds the embryo was hypertrophic in the groups treated with 0.12 and $0.5 \mathrm{mg}$ of LCM (Figures $1 \mathrm{~b}-\mathrm{d}$ ). Thus, different LCM treatment doses resulted in different effects (Figures $1 \mathrm{c}-\mathrm{f}$ ).

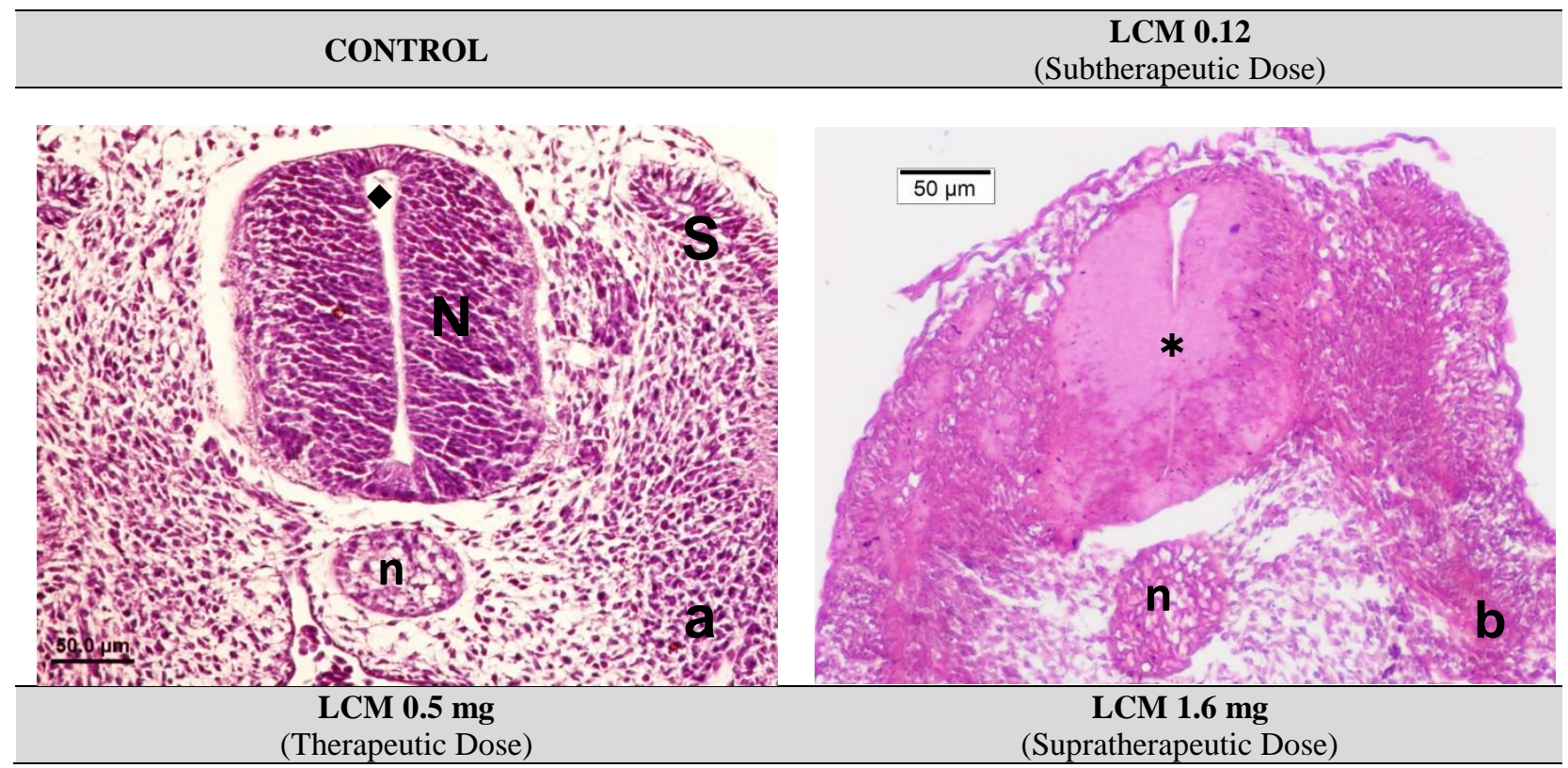



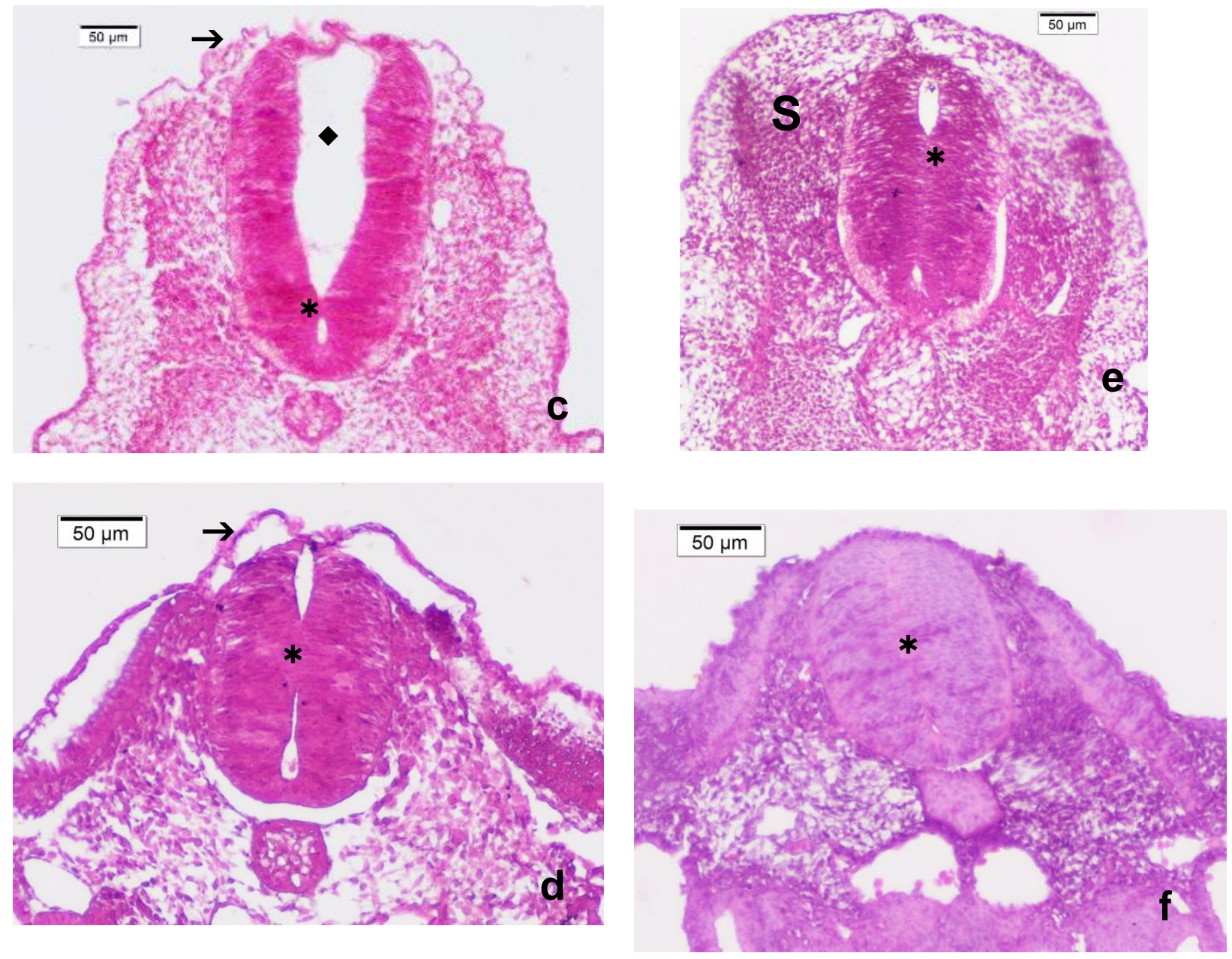

Figure 1. Control (a) and LCM-treated group (b-f) embryo sections. N: Neural Tube, $\bullet$ : Neural Cavity, n: Notochord, S: Somite, $\rightarrow$ : Hypertrophic Ectoderm, $*$ : Obstruction.

\section{Discussion}

Although epilepsy in pregnant women may cause serious non-invasive anomalies if no precautions are taken, the use of AEDs to control epilepsy can also cause fetal defects. For example, congenital abnormalities such as growth retardation, microcephaly, orofacial defects, and NT damage are associated with AED treatment. Mete et al. [6] investigated the effects of LCM on chicken embryo development and observed anencephaly with growth retardation during macroscopic examinations. The present study evaluated whether histological changes occurred at the NT level following LCM treatment and found that, although the NT closed, there were dosedependent increases in proliferating cells and pyknotic cells, and the cells were organized into a compact structure. At high LCM doses, the notochord was disrupted and hypertrophy was observed in the ectoderm layer surrounding the embryo.

Although AEDs markedly decrease the risks associated with epilepsy, a variety of symptoms, such as sedation, motor and behavioral changes, character changes, and disruptions in balance may occur [10]. Furthermore, the use of AED in critical stages of pregnancy increases the risk of these symptoms. For example, the presence of epilepsy in women prior to pregnancy, and the use of AEDs for long periods, increases the risk of neurotoxic effects in the fetus [11]. Although empirical and animal studies cannot perfectly mimic the human condition, these models are important for understanding the effects that might occur in humans. For example, animal studies have identified the presence of apoptotic cells in immature brains, decreased cell numbers in the hippocampus, hypothalamus, and cerebellum, and attenuated cell proliferation using these models [11-13]. Moreover, these studies suggested that the long-term effects associated with AEDs may include cognitive and behavioral defects. The present study observed apoptotic cells with a pyknotic morphology following LCM treatment, which is consistent with the literature [14]. 
The rate of congenital malformations in the general population is approximately $3 \%$ [15], which is similar to the rate in pregnant women who do not use AEDs; however, this risk increases by two- to threefold in mothers who use AEDs. One of the most common anomalies in this population is NTDs $[16,17]$ and the risk of minor congenital anomalies is threefold higher in this group versus the general population [18]. LCM is one of the newest members of the AED group and affects synaptic protein $2 \mathrm{~A}$, like other drugs in this class, by inhibiting neuronal voltage-gated sodium channels [19]. LCM also acts to disrupt the integrity of the cell skeleton [20]. AEDs generally produce neurotoxic effects during reflex maturation and can lead to cell death. In studies on chickens, high-dose AEDs cause growth anomalies and a combination of AED enhances these effects [6, 21].

Neuro-epithelium root cells form neurons, astrocytes, and oligodendrocytes throughout reproduction and cell differentiation in the NT, which occur at an earlier stage. At this stage, the effects of AEDs generally manifest as growth retardation in the caudal area and important changes are observed in affected glial cells [22]. Several changes also occur in neuroblast cells and AEDs have interactions with various cell types, particularly with those in the crista neuralis [22]. The changes associated with the use of AEDs progress in a parallel manner with intercellular adhesion models, as well as with changes in the extracellular matrix and growth factors [22].

Although the effects of AEDs can be defined as minor rather than major congenital anomalies at the NT level, they have the potential to cause serious problems in the future. The histological findings in the present study indicated that neurodevelopmental effects might onset over time; thus, further studies are needed to evaluate the long-term effects of AED, as well as the underlying mechanisms of action.

\section{References}

1. Unlu, A, Methods of developmental research, Acta neurochirurgica. Supplement, 2002, 83, 71-78.

2. Umur, AS, Selcuki, M, Bursali, A, Umur, N, Kara, B, Vatansever, HS, Duransoy, YK, Simultaneous folate intake may prevent adverse effect of valproic acid on neurulating nervous system, Child's nervous system : ChNS : official journal of the International Society for Pediatric Neurosurgery, 2012, 28(5), 729-737.

3. Kondo, A, Kamihira, O, Ozawa, H, Neural tube defects: prevalence, etiology and prevention, International journal of urology : official journal of the Japanese Urological Association, 2009, 16(1), 49-57.

4. Botto, LD, Moore, CA, Khoury, MJ, Erickson, JD, Neural-tube defects, The New England journal of medicine, 1999, 341(20), 1509-1519.
5. Pennell, PB, Use of Antiepileptic Drugs During Pregnancy: Evolving Concepts, Neurotherapeutics : the journal of the American Society for Experimental NeuroTherapeutics, 2016, 13(4), 811-820.

6. Mete, M, Gurcu, B, Collu, F, Unsal, UU, Duransoy, YK, Tuglu, MI, Selcuki, M, Effects of lacosamide "a novel antiepileptic drug" in the early stages of chicken embryo development, Child's nervous system : ChNS : official journal of the International Society for Pediatric Neurosurgery, 2016, 32(9), 1715-1719.

7. Guney, O, Canbilen, A, Konak, A, Acar, O, The effects of folic acid in the prevention of neural tube development defects caused by phenytoin in early chick embryos, Spine, 2003, 28(5), 442-445.

8. Hamburger, V, Hamilton, HL, A series of normal stages in the development of the chick embryo. 1951, Developmental dynamics : an official publication of the American Association of Anatomists, 1992, 195(4), 231-272.

9. Lindhout, D, Omtzigt, JG, Cornel, MC, Spectrum of neural-tube defects in 34 infants prenatally exposed to antiepileptic drugs, Neurology, 1992, 42(4 Suppl 5), 111-118.

10. Eddy, CM, Rickards, HE, Cavanna, AE, The cognitive impact of antiepileptic drugs, Therapeutic advances in neurological disorders, 2011, 4(6), . 385407.

11. Velez-Ruiz, NJ, Meador, KJ, Neurodevelopmental effects of fetal antiepileptic drug exposure, Drug safety, 2015, 38(3), 271-278.

12. Qiao, M, Malisza, KL, Del Bigio, MR, Kozlowski, $P$, Seshia, SS, Tuor, UI, Effect of long-term vigabatrin administration on the immature rat brain, Epilepsia, 2000, 41(6), 655-665.

13. Ikonomidou, C, Prenatal effects of antiepileptic drugs, Epilepsy currents, 2010, 10(2): p. 42-6.

14. Kellogg, M, Meador, KJ, Neurodevelopmental Effects of Antiepileptic Drugs, Neurochemical research, 2017, 42(7), 2065-2070.

15. Francine, R, Pascale, S, Aline1a, H, Congenital Anomalies: Prevalence and Risk Factors, Universal Journal of Public Health, 2014, 2, 58-63.

16. Guvenc, Y, Dalgic, A, Billur, D, Karaoglu, D, Aydin, S, Daglioglu, E, Ozdol, C, Nacar, OA, Yildirim, AE, Belen, D, The effects of levetiracetam on neural tube development in the early stage of chick embryos, Turkish neurosurgery, 2013, 23(5), 617-622. 
17. Hill, DS, Wlodarczyk, BJ, Palacios, AM, Finnell, RH, Teratogenic effects of antiepileptic drugs, Expert review of neurotherapeutics, 2010, 10(6), 943-959.

18. Lattanzi, S, Cagnetti, C, Foschi, N, Provinciali, L, Silvestrini, M, Lacosamide during pregnancy and breastfeeding, Neurologia i neurochirurgia polska, 2017, 51(3), 266-269.

19. Coppola, G, Piccorossi, A, Operto, FF, Verrotti, A, Anticonvulsant drugs for generalized tonic-clonic epilepsy, Expert opinion on pharmacotherapy, 2017, 18(9), 925-936.

20. Khanna, R, Wilson, SM, Brittain, JM, Weimer, J, Sultana, R, Butterfield, A, Hensley, K, Opening Pandora's jar: a primer on the putative roles of
CRMP2 in a panoply of neurodegenerative, sensory and motor neuron, and central disorders, Future neurology, 2012, 7(6), 749-771.

21. Ozgural, O, Armagan, E, Bozkurt, M, Eroglu, U, Kahilogullari, G, Unlu, A, The Effect of Levetiracetam on Closure of the Midline in Early Chicken Embryos, Turkish neurosurgery, 2015, 25(5), 681-684.

22. Ozer, FD, Demirel, A, Yilmaz Dilsiz, O, Aydin, M, Ozdemir, N, Uyanikgil, Y, Baka, M, Effects of Levetiracetam on neural tube development and closure of the chick embryos in ovo, Child's nervous system : ChNS : official journal of the International Society for Pediatric Neurosurgery, 2012, 28(7), 969976. 